\title{
Dysphagie
}

\section{Verschluckt! Ist doch halb so schlimm, oder?}

\author{
Nicht mehr sicher schlucken zu können, beeinträchtigt nicht nur die Lebensqualität vieler \\ älterer Patienten, sondern birgt auch potenziell tödliche Gefahren. Welche Maßnahmen \\ helfen können, erklärt Prof. Heppner in dieser Folge des consiliums Geriatrie.
}

\section{? Ich wurde in meiner Sprechstunde wiederholt von der Tochter einer älteren Patientin gefragt, ob sie die Me- dikamente für ihre Mutter nicht in Was- ser auflösen könnte, um Verschlucken zu verhindern. Ich würde dem Problem gern auf den Grund gehen. Welches sind die wichtigsten Schritte?}

Sie beschreiben hier ein häufiges und häufig übersehenes Problem. Schätzungen zufolge leiden etwa 5 Millionen Menschen an Schluckstörungen. Rund 14\% der selbstständig lebenden älteren Patienten, über 50\% der in Altenund Pflegeheimen und fast $70 \%$ der geriatrischen Krankenhauspatienten sind betroffen.

Bei einer Dysphagie gelangen Speisebrei, Flüssigkeiten und auch Medikamente versehentlich in die Luftröhre. Die Aspiration kann zu Erstickungsanfällen führen, oft aber auch still verlaufen und erst später zu schwerwiegenden Komplikationen führen. Oft geht sie mit einer Mangelernährung einher. Da Essen und Trinken integrale Bestandteile unserer sozialen Interaktion sind, führt die Dysphagie durch Angst vor dem Verschlucken überdies zum Verlust von Lebensqualität.

Die schwerwiegendste Komplikation der Dysphagie ist die Aspirationspneumonie. Es empfiehlt sich, schon beim geringsten Verdacht auf eine Schluckstörung einen Test in der Praxis durchzu-

\section{Mehr zum Thema}

Ein kostenloses Themenheft "Dysphagie“ von Prof. Dr. Dziewas und PD Dr. Wirth können Sie bei geriatrie@consilium.services anfordern.

\section{Tab. 1 Vorgehen bei Dysphagie}

\begin{tabular}{|l|l|}
\hline Problemstellung & Maßnahmen \\
\hline Mangelernährung erkennen & $\begin{array}{l}\text { Gewicht und Größe, BMI (eingeschränkt verwendbar), } \\
\text { Mini Nutritional Assessment (MNA), Ernährungsprotokoll }\end{array}$ \\
\hline Schluckstörung erkennen & $\begin{array}{l}\text { ScreeningTest, logopädische Beratung, } \\
\text { ggf. fiberendoskopische Schluckuntersuchung (FFES) }\end{array}$ \\
\hline Verordnung verändern & $\begin{array}{l}\text { Keine Mischkonsistenz, Andicken von Flüssigkeiten, } \\
\text { flüssig-orale Darreichungsformen bevorzugen }\end{array}$ \\
\hline
\end{tabular}

führen. Hierfür eignet sich die Beobachtung und Dokumentation beim Trinken eines Schlucks Wasser. Es wird kontrolliert, ob ein guter Lippenschluss erfolgt, ob auf zweimal geschluckt werden muss, ob Reste zurückbleiben und ob Husten oder eine belegte Stimme auftreten.

Das wesentliche Ziel ist die aspirationsfreie Ernährung und Medikamenteneinnahme. Dies bedeutet auch eine entsprechende Anleitung von Angehörigen oder Pflegenden durch eine logopädische Fachkraft. Flüssigkeiten sollten angedickt werden (modifizierte Maisstärke, Guarkernmehl). Wichtig ist, keine Mischkonsistenz (flüssig und fest) anzubieten (Suppe mit Einlage, Medikamentenkapsel und Wasser) und die Medikamenteneinnahme immer sitzend oder zumindest mit erhöhtem Oberkörper und ausreichend Flüssigkeit $(200 \mathrm{ml}$ Wasser) durchzuführen.

Alternative Darreichungsformen sind möglich, jedoch kommt es bei Mörsern, Auflösen etc. oftmals zu Veränderung der Wirkstofffreisetzung, weshalb eine enge Zusammenarbeit mit dem Apotheker nötig ist. Grundsätzlich sind bei Schluckbeschwerden oral-flüssige Arzneimittelformen bei der Darreichung zu bevorzugen.

\section{Zurück zum Fall}

Bei Ihrer Patientin sollten Sie zuerst einen einfachen Test durchführen, um eine Dysphagie zu erkennen. Wenn dieser positiv ausfällt ist, ist eine logopädische Fachuntersuchung anzuraten. Bei den Medikamenten sollte möglichst auf alternative Darreichungsformen umgestellt werden.

\section{$\rightarrow$ Anschrift des Verfassers:}

Univ.-Prof. Dr. med. H. J. Heppner, MHBA Klinik für Geriatrie HELIOS Klinikum Schwelm, Universität Witten/Herdecke Dr.-Moeller-Str. 15, D-58332 Schwelm E-Mail: hans.heppner@uni-wh.de

Haben Sie auch eine Frage an das consilium Geriatrie?

Haben Sie auch eine Frage, speziell aus den Bereichen Kognitive Störungen, Neurologie, Infektiologie, Schlafstörungen oder Dysphagie im Alter? Prof. Heppner und sein Expertenteam beantworten diese gerne. Das "consilium Geriatrie" ist ein gemeinsamer Service der MMW und des Unternehmens InfectoPharm.

Schicken Sie Ihre Frage kostenfrei an: geriatrie@consilium.services 